\title{
Eficácia dos métodos não farmacológicos para alívio da dor no trabalho de parto natural: uma revisão integrativa
}

\author{
Effectiveness of non-pharmacological methods for pain relief in natural labor: an \\ integrative review
}
Efectividad de los métodos no farmacológicos para el alivio del dolor en el trabajo de parto natural: una revisión integradora

Janaina Camilo de Freitas ${ }^{1 *}$, Cinderly Carvalho Silva ${ }^{1}$, Mariana Delfino Rodrigues ${ }^{1}$, Raquel Avelino Pereira de Souza 1 .

\section{RESUMO}

Objetivo: Identificar a eficácia dos métodos não farmacológicos para alívio da dor no trabalho de parto. Métodos: Trata-se de uma revisão integrativa da literatura de caráter exploratório e cunho qualitativo, foram utilizados os descritores "dor do parto", "trabalho de parto" e "terapias complementares" combinados entre si para a busca de artigos publicados no período de 2016 a 2021 na base de dados Biblioteca Virtual em Saúde (BVS), onde foram encontrados 74 artigos, após a utilização dos critérios de inclusão e exclusão, bem como análise rigorosa de conteúdo, resultando em uma amostra de 15 artigos. Resultados: Dentre a diversidade de métodos não farmacológicos para alívio da dor, verificou-se através desta revisão que o método mais frequentemente utilizado e com maiores índices de eficácia, foi o banho de aspersão. Considerações finais: Os métodos não farmacológicos para alívio da dor são variados, podendo ser ofertados tanto isoladamente quanto em conjunto, além de trazer benefícios também são de baixo custo, mostrando ser uma importante ferramenta para assistência às parturientes.

Palavras-chave: Dor do parto, Trabalho de parto, Terapias complementares.

\begin{abstract}
Objective: To identify the effectiveness of non-pharmacological methods for pain relief during labor. Methods: This is an integrative review of the literature of an exploratory and qualitative nature, using the descriptors "labor pain", "labor" and "complementary therapies combined with each other to search for articles published in the period of 2016 to 2021 in the VHL database, where 74 articles were found, after using the inclusion and exclusion criteria, as well as rigorous content analysis, resulting in a sample of 15 articles. Results: Among the diversity of non-pharmacological methods for pain relief, it was found through this review that the most frequently used method and with the highest rates of efficacy, was the sprinkler bath. Final considerations: The non-pharmacological methods for pain relief are varied and can be offered both individually and together, in addition to bringing benefits, they are also low-cost, proving to be an important tool for assisting parturients.
\end{abstract}

Key words: Labor pain, Labor obstetric, Complementary therapies.

\section{RESUMEN}

Objetivo: Identificar lá efectividad de los métodos no farmacológicos para aliviar el dolor en el trabajo de parto. Metodos: Esta es uma revisión integradora de la literatura de carácter exploratorio y cualitativo, utilizando los descriptores " el dolor del parto", " trabajo de parto" y " terapias complementarias" combinados entre sí para buscar artículos publicado en el período de 2016 a 2021 en la base de datos BVS donde fueron encontrados 74 artículos, después de utilizar los criterios de inclusión y exclusión, así como un análisis de contenido riguroso resultando en una muestra de 15 artículos. Resultados: Entre la diversidad de métodos no farmacológicos para aliviar el dolor se encontró mediante esta revisión que el método más utilizado y con mayores tasas de eficacia fue el baño de ducha. Consideraciones finales: Los métodos no farmacológicos para al alivio del dolor son variados y pueden ofrecerse tanto individuales como en conjunto, además de

\footnotetext{
${ }^{1}$ Centro Universitário Aparício Carvalho (FIMCA), Porto Velho - RO. *E-mail: janacamilo@outlook.com
} 
aportar beneficios, también son de bajo costo, demostrando ser una herramienta importante para ayudar a las mujeres parturientas.

Palabras clave: El dolor del parto, Trabajo de parto, Terapias complementarias.

\section{INTRODUÇÃO}

O período gestacional é um momento que requer cuidados especiais, pois resulta em diversas modificações, dentre elas: anatômicas, hormonais e psíquicas que afetam diretamente a saúde mental (VELHO MB, et al., 2014; LIMA MOP, et al., 2017). Essas mudanças mesmo que fisiológicas podem resultar em dores quer seja ainda no período gestacional ou no trabalho de parto (FERREIRA MLSM, et al., 2017). Sendo assim, é de suma importância a oferta de cuidados com objetivo de amenizar o processo de dor sem trazer riscos a mãe e ao bebê (LEHUGEUR D, et al., 2017).

Durante a gestação, principalmente primigestas podem criar um receio em relação à dor no trabalho de parto, e em decorrência disso somatiza o medo. Sabe-se que o estado emocional da gestante pode influenciar tanto positivamente como negativamente, por isso a importância da abordagem assistencial a ser prestada a essa mulher (MOTTA SAMF, et al., 2016).

Entre os fatores que ocasionam a dor nesse processo estão: a contração e dilatação do colo uterino, juntamente com o estiramento das fibras uterinas e a compressão na bexiga. Com isso, são conduzidos alguns manejos, tais como os métodos não farmacológicos para o alívio da dor. Para muitas puérperas essas técnicas, ajudam no relaxamento e proporcionando tranquilidade, desse modo, atua no alívio da dor (DIAS EG, et al., 2018; MASCARENHAS VHA, et al., 2019). Tendo em conta essas situações que resultam nas gestantes o sentimento de medo da dor do parto natural, o Ministério da Saúde (MS) criou condutas para ofertar atendimento humanizado através das Diretrizes Nacionais de Assistência ao Parto Normal (SANTOS ACM, et al., 2021).

A parturição normal com assistência humanizada é considerada a alternativa mais adequada de terapias complementares que visam a melhoria do atendimento com a parturiente e ao recém-nascido, onde o mesmo oferta o trabalho de parto menos doloroso e o vínculo entre mãe e filho. Esta assistência consiste em ofertar a gestante acolhimento, apoio, orientações e práticas de Métodos Não Farmacológicos que atuam no Alívio da Dor (MNFAD) do Trabalho de Parto (TP) natural (FERREIRA SV, et al., 2019; SANTOS ACM, et al., 2021).

Os MNFAD mais utilizados em maternidades são: banho de aspersão, bola suíça, massagens, presença de acompanhante, aromaterapia, musicoterapia, acupuntura, cavalinho, exercício de respiração, deambulação, entre outros (SOUZA AGN, et al., 2019). A aplicação destes MNFAD em todo o processo de nascimento assegura a gestante possuir mais autonomia sobre o processo de parturir (KATZER T, 2016). Essas condutas contribuem gerando diversas possibilidades de assistir a puérpera de acordo com sua necessidade (ALMEIDA CFS, et al., 2021).

Diante a diversidade de técnicas não farmacológicas, torna-se relevante a discussão da eficácia desses métodos, uma vez que podem contribuir positivamente no alívio da dor do trabalho de parto, aumentando o vínculo entre a mãe e o filho, diminuindo os danos, ocasionando uma rápida recuperação e ofertando uma assistência adequada as gestantes dentro dos padrões de humanização, respeitando e apoiando nesse processo natural da parturição. Diante dos pontos encontrados, o presente estudo teve como objetivo revisar e identificar a eficácia dos métodos não farmacológicos para alívio da dor no trabalho de parto.

\section{MÉTODOS}

Trata-se de um estudo realizado a partir de uma revisão integrativa da literatura, de caráter exploratório e de cunho qualitativo, sendo realizadas com base em pesquisas de artigos publicados no período de 2016 a 2021. A pesquisa partiu de uma pergunta-problema: "Qual a eficácia dos métodos não farmacológicos para alívio da dor no trabalho de parto natural?" 
O levantamento literário se deu inicialmente pela definição dos Descritores em Ciências da Saúde (DECS), aplicando os operadores booleanos para a pesquisa, sendo utilizado "AND" para combinar os descritores, resultando em "dor do parto AND terapias complementares", "trabalho de parto AND terapias complementares" e "dor do parto AND trabalho de parto. A busca pelos artigos ocorreu no período de janeiro a fevereiro de 2021, utilizando os descritores na base de dados Biblioteca Virtual em Saúde (BVS) Brasil.

Utilizou-se como critério de inclusão no estudo: artigos publicados entre os anos de 2016 a 2021; publicações em português; texto completo disponível na base de dados utilizada; estudos que abordassem a temática, incluindo dados referentes ao uso desses métodos. Os critérios para a exclusão foram: artigos que fogem do tema proposto e não tem relação com a pergunta norteadora; estudos com a metodologia de revisão, carta ao leitor, artigo de opinião, teses e dissertações; estudos publicados em outras plataformas. A Figura 1 demonstra o processo da seleção dos artigos com a utilização dos critérios de inclusão e exclusão.

Figura 1 - Fluxograma do processo de seleção dos artigos que compõem a amostra.

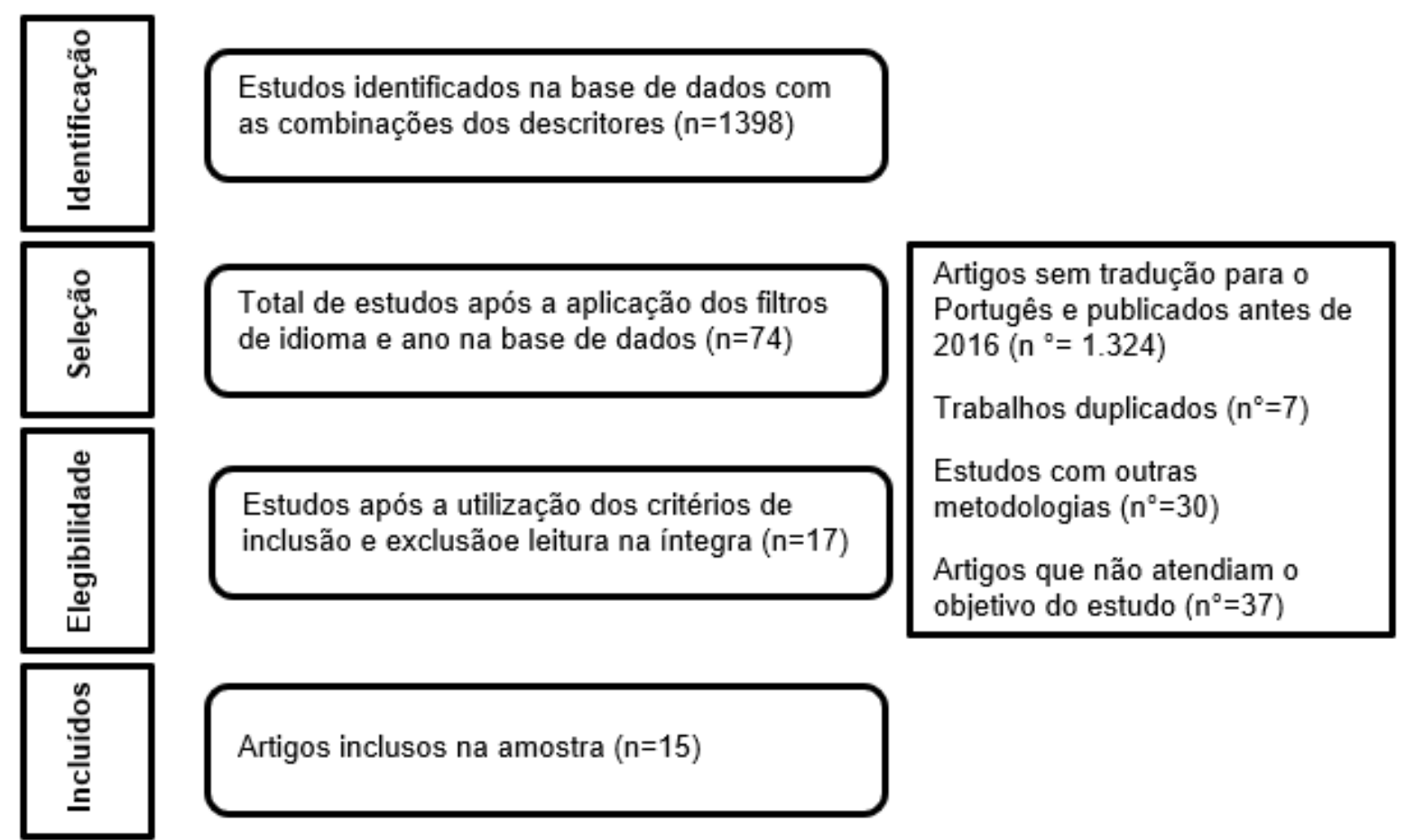

Fonte: Freitas JC, et al., 2021.

Ao realizar a busca na base de dados da BVS, utilizando a combinação dos descritores "dor do parto AND terapias complementares" obteve-se o resultado de 48 artigos, após a utilização dos filtros de idioma e dos últimos cinco anos, restou 10 artigos. Aplicando a combinação dos descritores "trabalho de parto AND terapias complementares", obteve-se 108 trabalhos, após a utilização dos filtros sobraram 13 artigos. Com a combinação de "dor do parto AND trabalho de parto" obteve-se 1.242 artigos, após a utilização dos filtros restou 51 artigos. Sendo assim, obteve-se 74 artigos, onde passaram por uma análise rigorosa de conteúdo, utilizando também os critérios de inclusão e exclusão e excluindo artigos duplicados, a amostra final resultou em 15 artigos.

\section{RESULTADOS E DISCUSSÃO}

Dos 15 artigos incluídos nessa revisão, dois (13\%) foram publicados no ano de 2016, dois (13\%) no ano de 2017, no ano de 2018 foram quatro (27\%) publicações, também publicados quatro (27\%) artigos em 2019 e três (20\%) em 2020. Sendo os anos de 2018 e 2019 os mais produtivos para publicações com essa temática. No Quadro 1 encontram-se os artigos divididos por título, autor, ano e resumo. 
Quadro 1 - Relação de artigos por título, autor, ano e resumo.

\begin{tabular}{|c|c|c|c|c|}
\hline $\mathbf{N}$ & Título & Autor & Ano & Resumo \\
\hline 1 & $\begin{array}{l}\text { Efeitos da acupressão sobre a dor no } \\
\text { trabalho de parto: ensaio clínico } \\
\text { randomizado. }\end{array}$ & $\begin{array}{l}\text { MAFETONI RR e SHIMO } \\
\text { AKK. }\end{array}$ & 2016 & $\begin{array}{l}\text { Os resultados indicaram que a acupressão no ponto BP6 atua no alívio da dor } \\
\text { de forma complementar, entretanto sugere que a redução causada é pequena } \\
\text { e que pode tornar-se mais eficaz se utilizada com dilatação } 8 \mathrm{~cm} \text { e apresentação } \\
\text { cefálica. }\end{array}$ \\
\hline 2 & $\begin{array}{l}\text { Hidroterapia e bola suíça no trabalho } \\
\text { de parto: ensaio clínico randomizado. }\end{array}$ & HENRIQUE AJ, et al. & 2016 & $\begin{array}{l}\text { O artigo mostrou que quando ambos os manejos combinados a eficiência e } \\
\text { eficácia são ainda mais efetivos, resultando pontos positivos. }\end{array}$ \\
\hline 3 & $\begin{array}{l}\text { Estratégias não farmacológicas para } \\
\text { o alívio da dor no trabalho de parto: } \\
\text { efetividade sob a ótica da parturiente. }\end{array}$ & HANUM SP, et al. & 2017 & $\begin{array}{l}\text { O artigo mostra que há falta de informação no período gravídico. O estudo } \\
\text { aponta que o melhor e mais utilizado entre elas foi o método de banho morno, } \\
\text { como também muito utilizados os exercícios respiratórios de relaxamento e a } \\
\text { massagem lombossacral. }\end{array}$ \\
\hline 4 & $\begin{array}{l}\text { Manejo não farmacológico de alívio } \\
\text { da dor em partos assistidos por } \\
\text { enfermeira obstétrica. }\end{array}$ & LEHUGEUR D, et al. & 2017 & $\begin{array}{l}\text { O presente estudo relata sobre partos assistidos por enfermeira obstétrica } \\
\text { quanto aos manejos não farmacológicos para alívio da dor, resultando } 98,3 \% \\
\text { utilizaram algum método não farmacológico de alívio da dor, a saber: } \\
\text { deambulação }(79,2 \%) \text {, banho }(73,1 \%) \text {, massagem }(60,0 \%) \text {, variedade de } \\
\text { posição }(58,8 \%) \text {, aromaterapia }(46,9 \%) \text {, bola }(42 \%) \text {, entre outros. }\end{array}$ \\
\hline 5 & $\begin{array}{l}\text { Efeitos da auriculoterapia no tempo } \\
\text { de trabalho de parto e taxa de } \\
\text { cesárea: ensaio clínico randomizado. }\end{array}$ & MAFETONI RR, et al. & 2018 & $\begin{array}{l}\text { Nesta pesquisa, a média de duração do trabalho de parto foi menor no grupo } \\
\text { de auriculoterapia }(607,8 \text { versus placebo: } 867,9 \text { versus controle: } 694,7 \text { minutos; } \\
\text { p-valor }=0,845) \text {. }\end{array}$ \\
\hline 6 & $\begin{array}{l}\text { Tecnologias não invasivas de } \\
\text { cuidado: percepção das puérperas. }\end{array}$ & PEREIRA PSL, et al. & 2018 & $\begin{array}{l}\text { Houve satisfação em relação aos cuidados prestados aliviando a dor, } \\
\text { proporcionando bem-estar e diminuindo o tempo de espera durante o trabalho } \\
\text { de parto. Esses cuidados oferecidos pelos profissionais, em sua grande maioria, } \\
\text { foram avaliados positivamente pelas participantes deste estudo. }\end{array}$ \\
\hline 7 & $\begin{array}{l}\text { Representações de puérperas sobre } \\
\text { os cuidados recebidos no trabalho de } \\
\text { parto e parto. }\end{array}$ & MELO LPT, et al. & 2018 & $\begin{array}{l}\text { A partir da análise realizada pode-se perceber que as palavras dor, felicidade e } \\
\text { orientação apareceram com mais frequência como termos indutores. Dessa } \\
\text { forma, conclui-se que o momento do trabalho de parto e do parto é crucial para } \\
\text { que o enfermeiro planeje e execute uma adequada assistência durante o } \\
\text { processo de parturição. }\end{array}$ \\
\hline 8 & $\begin{array}{l}\text { Eficiência de métodos não } \\
\text { farmacológicos para alívio da dor no } \\
\text { trabalho de parto normal. }\end{array}$ & DIAS EG, et al. & 2018 & $\begin{array}{l}\text { O banho de aspersão foi o método mais utilizado. Os métodos foram eficazes } \\
\text { quanto a sua finalidade e associaram a eles sentimentos de satisfação, } \\
\text { relaxamento e tranquilidade. O acompanhante e o profissional de saúde } \\
\text { apareceram como estratégia de suporte para efetivação dos métodos usados. }\end{array}$ \\
\hline
\end{tabular}




\begin{tabular}{|c|c|c|c|c|}
\hline $\mathbf{N}$ & Título & Autor & Ano & Resumo \\
\hline 9 & $\begin{array}{l}\text { Conhecimento e aplicabilidade dos } \\
\text { métodos não farmacológicos } \\
\text { utilizados pelos enfermeiros } \\
\text { obstetras para alívio da dor no } \\
\text { trabalho de parto. }\end{array}$ & CAMACHO ENPR, et al. & 2019 & $\begin{array}{l}\text { Os métodos mais citados entre eles foram: massagem sacrolombar, banho de } \\
\text { imersão, bola suíça, deambulação, cavalinho, exercícios de respiração, entre } \\
\text { outros. Em relação ao conhecimento das puérperas, uma pequena parcela } \\
\text { demonstrou conhecimento, e a aplicabilidade, o estudo mostra que ainda há } \\
\text { falta e dificuldade da prática. }\end{array}$ \\
\hline 10 & $\begin{array}{l}\text { A prática de métodos não } \\
\text { farmacológicos para o alívio da dor de } \\
\text { parto em um hospital universitário no } \\
\text { Brasil. }\end{array}$ & MIELKE KC, et al. & 2019 & $\begin{array}{l}\text { Concluiu-se que o manejo mais eficaz é o banho quente, pois além de baixo } \\
\text { custo, não-invasivo, favorece o relaxamento da musculatura, ocasionando } \\
\text { assim o alívio da dor. }\end{array}$ \\
\hline 11 & $\begin{array}{l}\text { Conhecimento das gestantes acerca } \\
\text { das medidas de alívio da dor durante } \\
\text { o parto. }\end{array}$ & SILVA AS, et al. & 2019 & $\begin{array}{l}\text { Objetivou-se em analisar os conhecimentos das puérperas em seu primeiro } \\
\text { parto acerca dos métodos não farmacológicos, foram ao todo } 49 \text { participantes } \\
\text { e } 100 \% \text { afirmaram não ter tido oferta desses manejos durante o pré-natal, } \\
\text { somente durante o trabalho de parto e mesmo assim que o nível de } \\
\text { conhecimento foi baixo. }\end{array}$ \\
\hline 12 & $\begin{array}{l}\text { Terapias complementares no } \\
\text { trabalho de parto: ensaio clínico } \\
\text { randomizado. }\end{array}$ & CAVALCANTI ACV, et al. & 2019 & $\begin{array}{l}\text { Foram utilizadas as terapias de banho quente e bola suíça, onde as mesmas } \\
\text { não interferiram na redução da dor durante o trabalho de parto, entretanto todas } \\
\text { demostraram efeito positivo no que se refere a abreviação do tempo de } \\
\text { evolução do trabalho de parto ao nascimento, especialmente quando utilizadas } \\
\text { de forma combinada. }\end{array}$ \\
\hline 13 & $\begin{array}{l}\text { Parâmetros maternos e perinatais } \\
\text { após intervenções r não } \\
\text { farmacológicas: um ensaio clínico } \\
\text { randomizado controlado. }\end{array}$ & MELO PS, et al. & 2020 & $\begin{array}{l}\text { O banho quente isolado ou em combinação com exercícios perineais com bola } \\
\text { suíça resultou em aumento no número de contrações uterinas em comparação } \\
\text { com somente a intervenção com bola suíça. }\end{array}$ \\
\hline 14 & $\begin{array}{l}\text { Tecnologias de cuidado para o alívio } \\
\text { da dor na parturição. }\end{array}$ & MARINS RB, et al. & 2020 & $\begin{array}{l}\text { No grupo utilizado para amostra descreveu como excelente e de grandes } \\
\text { vantagens as tecnologias que se utilizam para alívio da dor do trabalho de parto. } \\
\text { Portanto, fica evidente a importância do uso desses manejos para uma vivência } \\
\text { positiva do parto, mais prazerosa e menos traumatizante. }\end{array}$ \\
\hline 15 & $\begin{array}{l}\text { Vivências de mulheres em trabalho } \\
\text { de parto com o uso de essências } \\
\text { florais. }\end{array}$ & DE LARA SRG, et al. & 2020 & $\begin{array}{l}\text { Evidenciou que os efeitos da terapia floral, atuaram em sinergia, na redução } \\
\text { dos sintomas de estresse-medo-tensão, além do aumento do bem-estar } \\
\text { emocional proporcionando às parturientes a oportunidade de protagonizar o seu } \\
\text { próprio trabalho de parto e parto. }\end{array}$ \\
\hline
\end{tabular}

Fonte: Freitas JC, et al., 2021. 
Dentre a diversidade de métodos não farmacológicos, verificou-se através desta revisão que método mais frequentemente utilizado foi o banho de aspersão. Dias EG, et al. (2018) verificou que durante a aplicação do banho de aspersão, as parturientes relataram alívio na tensão, redução da dor e proporcionando mais tranquilidade, além disso trata-se de um método de fácil aplicabilidade.

Corroborando com Dias EG, et al. (2018), Cavalcanti ACV, et al. (2019) revelam que o banho quente resulta na redução da ansiedade. Nesse mesmo estudo, os autores verificaram que a associação do banho quente, o uso da bolsa suíça e exercícios perineais resultou em um aumento da dor relatada pelas parturientes, no entanto mesmo sem o alívio da dor foi percebido uma melhoria do bem estar, refletindo que mesmo sem o alívio da dor, essas terapias proporcionaram um melhor enfrentamento da mesma.

Melo PS, et al. (2020) constataram que os MNFAD de forma isolada ou combinadas são seguros e eficazes. As contrações uterinas e dilatação cervical aumentaram com o uso de banho quente, tendo o mesmo resultado quando associado a exercícios perineais, já em relação às contrações não houve grande diferenciação, entretanto, a duração foi menor para as que realizaram o banho de imersão. Os exercícios perineais com a bola suíça trouxeram benefícios em relação ao tempo, uma vez que foi identificado um encurtamento no tempo de trabalho parto, como também, auxiliou na descida e estação da cabeça do bebê na pelve materna. Dessa forma, o uso da bola suíça segundo os autores deve ser incentivado pelos profissionais durante a assistência, pois auxilia de forma positiva no processo de parturição.

Henrique AJ, et al. (2016) evidenciaram que os métodos de banho morno e bola suíça quando associados possuem uma melhor eficácia, verificou-se que as mulheres que utilizam ambas as técnicas obtiveram uma descida da apresentação fetal mais rápida, melhor evolução da dilatação cervical, menor uso de analgesias, menor tempo no trabalho de parto e a taxa de parto cesárea foi mais baixa comparadas as que utilizaram esses manejos isoladamente.

O banho morno e a bola suíça são intervenções de baixo custo e de fácil acesso, também há uma boa aceitação por parte das gestantes, não traz riscos para a parturiente e nem para o bebê, há maior eficácia quando esses métodos são utilizados associadamente (HENRIQUE AJ, et al., 2016).

A utilização desses métodos associados é de grande vantagem, sendo necessários mais estudos que abordem a contribuição para a redução de parto cesárea, uma vez que se pode colaborar na diminuição em casos que não há necessidade para esse tipo de parto, por serem também métodos acessíveis e por proporcionar um TP menos doloroso para a parturiente.

Outro método não farmacológico encontrado foi os exercícios respiratórios, um método de baixo custo e acessível á todas as parturientes, não exige uma atenção acentuada dos profissionais e pode ser realizada com auxílio do acompanhante. As orientações devem-se concentrar na forma de inspiração e expiração pausadamente durante as contrações (CAMACHO ENPR, et al., 2019).

Os exercícios respiratórios têm como objetivo controlar a ansiedade, e aumentar a oxigenação da parturiente e do feto, com isso promove relaxamento, diminuindo o desconforto do trabalho de parto, além de que aumenta a pontuação de Aparência, Pulso, Careta, Atividade e Respiração (APGAR) no primeiro minuto após o parto (PEREIRA PSL, et al., 2020).

Camacho ENPR, et al. (2019), afirma que os exercícios respiratórios promovem a diminuição de riscos de trauma perineal no momento da expulsão do bebê, uma concentração maior da parturiente neste momento delicado, e quando ofertados há uma redução significativa da dor no trabalho de parto, porém podem não ser o suficiente para reduzir a dor do parto, com isso é aconselhável ser combinado com algum outro manejo.

Segundo Hanum SP, et al. (2017), considerando a particularidade de cada parturiente, os exercícios respiratórios só apresentam eficácia no alívio da dor quando combinado com algum outro método não farmacológico. Encontrado também como MNFAD, a posição do parto que de acordo com Lehugeur $\mathrm{D}$, et al. (2017), alternar a posição litotômica, para outras posições oferta alívio da dor através do aumento da velocidade da dilatação cervical, dependendo da posição, facilita a descida fetal e assim diminui a dor do parto. 
Pereira PSL, et al. (2018), afirmam que apesar da parturiente ter tido bom preparo para o parto, ainda há déficit de conhecimento desse método, assim como a equipe de profissionais também não buscam ofertar a mulher este serviço, fazendo assim um retrocesso do trabalho de parto. Ele também traz em seu artigo o uso da banqueta de meia lua como um MNFAD, mas o mesmo afirma que não há evidências comprovando o alívio da dor, mas sim relatos deixando explícito que o tempo do trabalho encurta, e com isso faz com que sinta dor em menos tempo.

Já segundo Silva AS, et al. (2019), não é possível afirmar se este método é eficaz ou não, pois o déficit no conhecimento das parturientes é alto, de acordo com a sua pesquisa de 49 gestantes, somente $6,1 \%$ tinha conhecimento da variação da posição do parto. Portanto, vemos que por esta falta de conhecimento fica limitada a oferta do manejo e assim sem resultado concreto se alivia a dor do trabalho de parto e parto.

A massagem apresenta eficácia no alívio da dor, pois atua na estimulação sensorial, ativando o fluxo sanguíneo e gerando o relaxamento muscular, ainda sim reduz o estresse. Pode ser realizada no local onde a parturiente está acusando dor, ou com auxílio de dispositivos como a bola suíça, onde a massagem ocorrerá em região perineal (LEHUGEUR D, et al., 2017; DIAS EG, et al., 2018).

Dias EG, et al. (2018), apontaram em seu estudo que as puérperas que utilizaram da massagem apresentam satisfação, pois alivia a dor, proporciona o bem-estar, a equipe de profissionais responsável também é mais disposta a ofertar a este manejo. Em contrapartida, Camacho ENPR, et al. (2019) afirmam que a oferta da massagem é algo muito relativo, pois a sua execução requer tempo e disponibilidade dos profissionais, visto que além da assistência os mesmos também são responsáveis pelas atividades burocráticas. Apesar de não existir comprovações relacionadas aos riscos e benefícios da massagem, sabese que quando utilizada o TP torna-se um momento agradável e satisfatório.

O direito ao acompanhante também é integrado aos MNFAD, pois além de ser visto como um suporte afetivo faz com que a gestante no seu momento de parturição sinta-se apoiada, encorajada, segura, também auxilia para a redução da ansiedade e com isso traz uma sensação de tranquilidade, tornando assim o momento mais confortável (DIAS EG, et al., 2018; PEREIRA PSL, et al., 2018; CAVALCANTI ACV, et al., 2019).

O bem-estar materno contribui para uma evolução tranquila do trabalho de parto, $100 \%$ das mulheres que tiveram seu parceiro ou acompanhante presente nesse momento obtiveram avanço significativo (LEHUGEUR D, et al., 2017; CAVALCANTI ACV, et al., 2019). O acompanhante torna o ambiente agradável a mulher, favorecendo o bem-estar emocional e físico, fazendo-se assim a puérpera obter uma boa evolução do período gravídico-puerperal (HANUM SP, et al., 2017).

O parto é um momento muito importante e único, o cuidado humanizado é primordial, juntamente com o auxílio dos métodos não farmacológicos para proporcionar uma boa experiência a essa mulher. Dias EG, et al. (2018) mostram a importância da participação dos profissionais de saúde para o encorajamento e apoio emocional as parturientes, podendo ajudar na redução do medo e alívio da dor.

Melo LPT, et al. (2018) afirma que além dos fatores físicos, os socioculturais e psicológicos interferem diretamente no processo de parturição. Diante disso, cabe a equipe prestar esse suporte a gestante e seu companheiro, desmistificando, quebrando tabus e preconceitos em relação ao momento do parto. Proporcionando uma assistência humanizada e de qualidade.

Medidas intervencionista desnecessárias atrapalham o protagonismo da gestante nesse processo tão importante, as técnicas quando aplicadas com estratégias trazem como resultado alívio considerado da dor no trabalho de parto. Grande parte dos estudos sugere pesquisas com estratégias que auxiliem quanto a orientações e nas práticas dos MNFAD (MARINS RB, et al., 2020). De acordo com Camacho ENPR, et al. (2019), a deambulação apesar de favorecer o trabalho de parto, é pouco utilizado pois muitas parturientes fazem uso de ocitocina dificultando a movimentação das mesmas.

Segundo Lehugeur D, et al. (2017) e Mielke KC, et al. (2019), a deambulação aumenta a velocidade do TP, pois a mobilidade pélvica aumenta o fluxo sanguíneo, consequentemente proporciona uma melhoria da 
oxigenação dos tecidos que somada a posição vertical faz com que a gravidade atue na descida do feto, ativando a dilatação e aumentando a frequência das contrações uterinas. Em contrapartida, o mesmo mecanismo que alivia a dor, pode gerar à gestante um reflexo de intensificação da dor visto ao menor espaço de tempo das contrações, assim é um método a ser usado de forma combinada para melhor eficácia. Portanto, a deambulação torna-se um método não farmacológico que alivia a dor do trabalho de parto utilizada de forma positiva nas parturientes.

Estudos realizados por De Lara SRG, et al. (2020) e Lehugur D, et al. (2017) revelaram que o uso de essências florais proporcionou as parturientes a sensação de relaxamento e calmaria, ajudando a reduzir o medo e estresse. Esse conjunto de sentimentos trouxe como consequência um auxílio no controle da dor. Os óleos essenciais podem ser utilizados para reduzir a dor, sendo absorvidos por meio de inalação ou uso tópico na pele. Entretanto, pode-se observar que há necessidade de pesquisas direcionadas a aromaterapia, uma vez que dentre os artigos, apenas dois estudos abordaram este método.

Observou-se a necessidade de ações voltadas para a saúde da mulher durante o pré-natal, pois há grande deficiência de informações sobre os MNFAD. Através dessas orientações pode-se evitar experiências negativas no momento do parto. Da mesma forma, os profissionais devem ter sensibilidade e estar sempre atentos para suprir e sanar dúvidas relacionadas a temática, trazendo orientações sempre que necessário (SILVA AS, et al., 2019; HANUM SP, et al., 2017).

O cavalinho é em um assento onde a puérpera encontra-se numa posição levemente inclinada para frente com apoio para os braços e costas. Ampliando a pelve deixando a região sacrolombar livre, favorecendo a rotação do bebê e o conforto promovido torna as contrações uterinas menos dolorosas (PEREIRA PSL, et al., 2018). Porém Hanum SP, et al. (2017) traz em seu artigo que o cavalinho quando comparado a outros manejos, mostra-se não eficaz, pois em seu estudo ele trouxe menos $5 \%$ de eficácia, de acordo com os relatos das parturientes na sua pesquisa.

Das medidas não farmacológicas para o alívio da dor, verificou-se que dentre os artigos incluídos nessa revisão, apenas 1 abordou a utilização da auriculoterapia para o alívio da dor no trabalho de parto. Mafetoni $\mathrm{RR}$, et al. (2018) mostram que as parturientes que utilizaram esse método obtiveram um menor tempo na duração do trabalho de parto quando comparado às que não fizeram o uso. Assim, verifica-se a importância de novos estudos demonstrando a eficácia. Vale ressaltar que a acupuntura está descrita na Política Nacional de Práticas Integrativas Complementares no SUS.

Dentre os artigos, apenas 1 abordou a acupressão como manejo não farmacológico para o alívio da dor no trabalho de parto. Mafetoni RR e Shimo AKK (2016) demonstraram que houve uma redução no alívio da dor, obtendo um melhor resultado quando aplicados em parturientes com dilatação a partir de 8 centímetros, também observou-se uma evolução mais rápida no trabalho de parto daquelas que utilizaram o manejo.

O estudo demonstrou que a acupressão é de baixo custo, fácil acesso e que não traz malefícios a parturientes e nem ao recém-nascido, mostrou também a falta de informação das gestantes sobre esse método (MAFETONI RR e SHIMO AKK, 2016).

\section{CONSIDERAÇÕES FINAIS}

Conforme os dados apresentados, pode-se afirmar que os métodos não farmacológicos são eficazes e trazem diversos benefícios no trabalho de parto. Em relação ao alívio da dor, alguns estudos mostram que ele auxilia em outros aspectos fazendo com que o relaxamento leve a sensação de alívio da dor, já outros revelam que as parturientes obtiveram alívio da dor utilizando esses manejos, entretanto, tendo em vista a subjetividade da dor em cada parturiente, sendo ela imensurável, ainda é necessário que haja mais estudos explorando os métodos separados de acordo com as fases do TP e sua relação direta na atuação para alívio da dor. 


\section{REFERÊNCIAS}

1. ALMEIDA CFS, et al. Métodos alternativos para alívio da dor no parto normal: revisão sistemática. Research, Society and Development, 2021; 10(1).

2. CAMACHO ENPR, et al. Conhecimento e aplicabilidade dos métodos não farmacológicos utilizados pelos enfermeiros obstetras para alívio da dor no trabalho de parto. Revista Nursing, 2019; 22, (257).

3. CAVALCANTI ACV, et al. Terapias complementares no trabalho de parto: ensaio clínico randomizado. Revista gaúcha de enfermagem, 2019; 40.

4. DE LARA SRG, et al. Vivência de mulheres em trabalho de parto com o uso de essências florais. Reviste online de pesquisa, 2020; 20.

5. DE SOUZA MT, et al. Revisão integrativa: o que é e como fazer. Einstein, 2010; 1(8).

6. DIAS EG, et al. Eficiência de métodos não farmacológicos para alívio da dor no trabalho de parto normal. Revista cofen, 2018; 9(2).

7. FERREIRA MLSM, et al. Dor como motivo de busca para assistência ao trabalho de parto hospitalar. Revista Eletrônica de Enfermagem, 2017.

8. FERREIRA SV, et al. Cuidado de enfermagem na óptica das gestantes de alto risco. Revista Família, Ciclos de vida e saúde no Contexto Social, 2019; 7(2).

9. GERHARDT T; SILVEIRA, D. Métodos de pesquisa. Editora da UFRGS, 2009.

10. HANUM SP, et al. Estratégias não farmacológicas para alívio da dor no trabalho de parto: efetividade sob a ótica da parturiente. Revista de Enfermagem UFPE on line, 2017; 11(8).

11. HENRIQUE AJ, et al. Hidroterapia e bola suíça no trabalho de parto: ensaio clínico randomizado. ACTA Paulista de Enfermagem, 2016; 29(6).

12. KATZER T. Métodos não farmacológicos para o alívio da dor: percepções da equipe multiprofissional no trabalho de parto e parto. Dissertação. Santa Cruz do Sul, 2016.

13. LEHUGEUR D, et al. Manejo não Farmacológico da Dor em Partos Assistidos por Enfermeira Obstétrica. Revista de Enfermagem UFPE, 2017; 11(12)..

14. LIMA MOP et al. Sintomas depressivos na gestação e fatores associados: estudo longitudinal. Rede de Revistas Cientificas da América Latina, Caribe - Redalyc. Acta Paulista de Enfermagem, 2017; 30(1).

15. MAFETONI RR, et al. Efeitos da auriculoterapia no tempo de trabalho de parto e taxa de cesárea: ensaio clínico randomizado. REME, 2018; 22.

16. MAFETONI RR; SHIMO AKK. Efeitos da Acupressão sobre a dor no trabalho de parto. Rev Latino-Americano de Enf. Ribeirão Preto 2016; 24.

17. MARINS RB, et al. Tecnologias de cuidado para o alívio da dor na parturição. Revista FunCare online, $2020 ; 12$.

18. MASCARENHAS VHA, et al. Evidências científicas sobre métodos não farmacológicos para alívio da dor do parto. ACTA Paulista de Enfermagem, 2019; 32(3).

19. MELO LPT, et al. Representações de puérperas sobre os cuidados recebidos no trabalho de parto e parto. Av Enferm, 2018; 36(1).

20. MELO PS, et al. Parâmetros maternos e perinatais após intervenções não farmacológicas: um ensaio clínico randomizado controlado. Revista Acta Paul Enfermagem, 2020; 33.

21. MIELKE KC, et al. A prática de métodos não farmacológicos para o alívio da dor de parto em um hospital universitário no Brasil. Avances em Enfermagem, 2019; 37(1).

22. MOTTA SAMF, et al. Implementação da Humanização da Assistência ao parto natural. Revista de Enfermagem UFPE, 2016; 2(10).

23. OLIVEIRA MC, et al. Metodologias de Pesquisas Adotadas nos Estudos Sobre Balanced Scorecard. Congresso Brasileiro de Custos, 2006.

24. PEREIRA PSL, et al. Tecnologias não invasivas de cuidado: percepção das puérperas. Revista de Enfermagem UFPE, 2018; 12(8).

25. SANTOS ACM, et al. Atuação da enfermagem no uso de métodos não farmacológicos para o alívio da dor durante o trabalho de parto. Brazilian Journal of Development, 2021; 7(1).

26. SILVA AS, et al. Conhecimento das gestantes acerca das medidas de alívio da dor durante o parto. Revista enfermagem atual, 2019; 87(25).

27. SOUZA AGN, et al. Avaliação dos métodos não farmacológicos para alívio da dor (MNFAD) no trabalho de parto por puérperas. Revista Eletrônica Acervo Saúde, 2020; 50.

28. VELHO MB, et al. Parto normal e cesárea: representações sociais de mulheres que vivenciaram. Revista Brasileira de Enfermagem (ReBEn), 2014; 67(2). 